\title{
MOTION OF AN EXPLOSIVE-INDUCED PLANE SHOCK WAVE*
}

BY

\author{
W. FICKETT
}

Los Alamos Scientific Laboratory

\begin{abstract}
A plane, unsupported, Chapman-Jouguet detonation in a condensed explosive drives a decelerating shock into a semi-infinite inert of lower shock impedance. A previously reported exact solution for a portion of this one-dimensional time-dependent problem is extended to the entire flow field, and some numerical results are given. The solution has the form of a small set of first-order ordinary differential equations for the shock, and a similar set for each particle path.
\end{abstract}

1. Introduction. This one-dimensional time-dependent problem of the decelerating shock in a semi-infinite inert material driven by a finite length of explosive is fundamental in explosives work. The physical approximations are as follows. The chemical reaction is assumed to be instantaneous, so that we have to deal only with the reaction products, which we refer to as "the explosive". Both the explosive and the inert are treated as inviscid fluids, so that the Euler equations of compressible flow apply.

Approximate solutions of this problem have been given [1, 2]. By proper choice of special but quite realistic equations of state for the two materials, the partial differential equations describing the motion may be reduced with no approximation to a set of ordinary differential equations with one independent variable. Solutions of this type for the motion of the interface and shock have been given by Kondratev et al. [3]. We here extend the exact solution to the entire flow field. Having in mind as an important application the testing of computer programs which solve the partial differential equations of motion by finite-difference methods, we give all the final equations in a form having time as the convenient independent variable, and furnish a table of numerical results. We also describe, for the relatively complicated inert equation of state, a tested computational procedure suitable for use in a finite-difference computer program.

For background, and for standard results used here without explicit reference, the reader is referred to [1-4].

The problem is described in Sec. 2, the equations of state in Sec. 3 and in the Appendix, the solution in Sec. 4, and numerical results in Sec. 5.

2. The problem. The body (Fig. 1) consists of a finite length of condensed explosive driving a semi-infinite inert. The flow is assumed to be one-dimensional, that is, all

* Received October 2, 1972; revised version received March 23, 1973. Work performed under the auspices of the U. S. Atomic Energy Commission. The author would like to thank W. C. Davis, W. C. Rivard and W. W. Wood for helpful conversations and suggestions, W. C. Rivard and R. P. Engelke for a careful reading of the manuscript, and D. Dickman for performing some of the caluclations. 


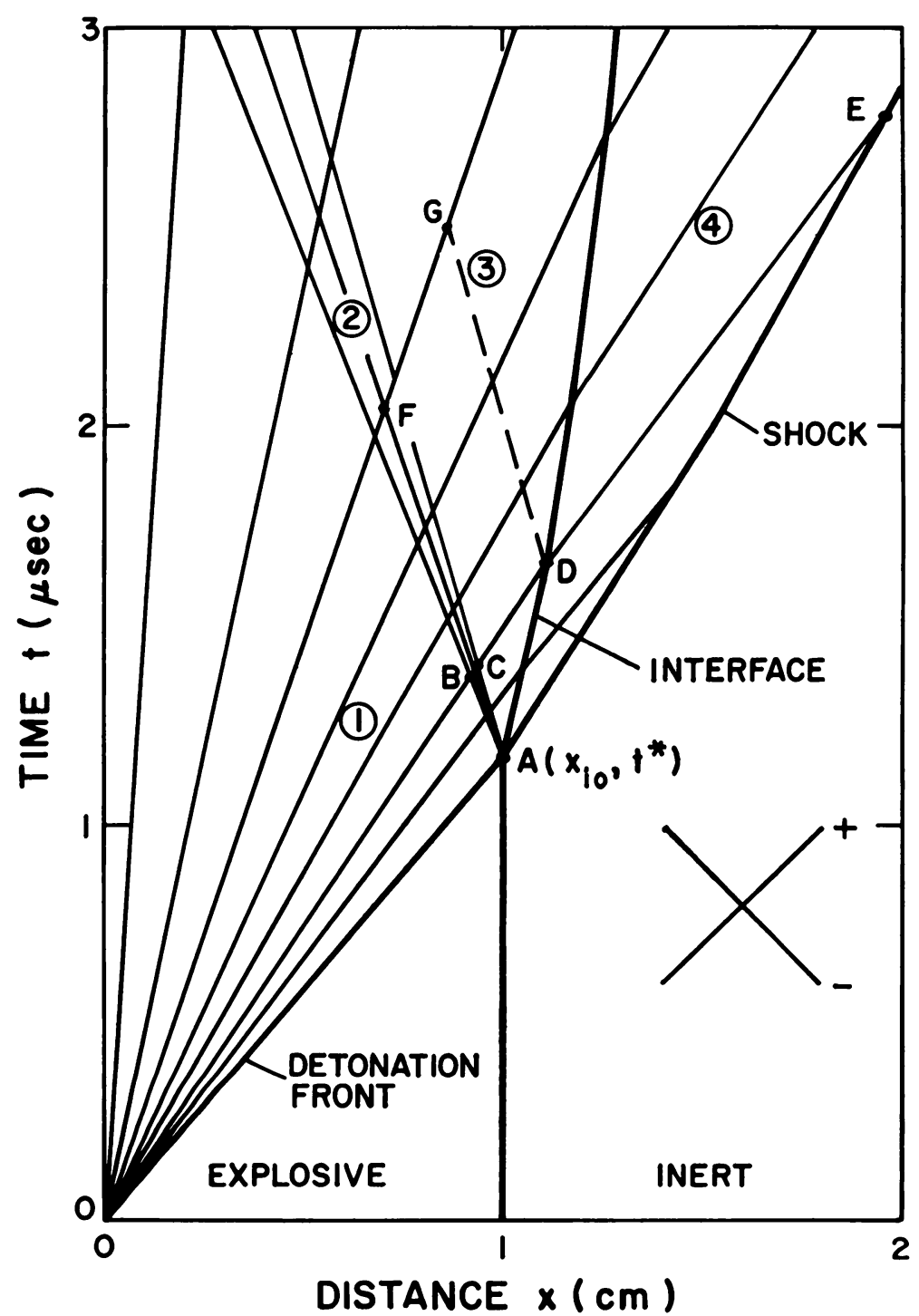

Fig. 1. The $x-t$ diagram of the motion. The explosive is Composition $B$ and the inert is plexiglas.

dependent variables are functions only of $x$ and $t$. The initial condition at $t=0$ is that both materials have their uniform normal density at $p=0$. The left boundary condition is $p=0$. The detonation is initiated at the left boundary at time $t=0$. Under our assumptions the detonation front consists of a shock in which the chemical reaction is completed instantaneously. This is followed by a right-facing rarefaction wave, the so-called Taylor wave (region 1 of the figure). When the detonation front reaches the interface, a centered left-facing rarefaction (region 2) is reflected back into the explosive, and a shock is transmitted into the inert. The Taylor wave passes through the left-facing centered rarefaction into region 3 , where it interacts with its own partial reflection from the interface. The transmitted shock is followed by the transmitted Taylor rarefaction 
region 4) which continually reduces its strength. The expansion of the reaction products to the left of $x=0$ is not shown in the figure. Fig. 2 is the $p-u$ diagram and Fig. 3 a sketch of the pressure profile at time $t=2 \mu \mathrm{s}$.

We use the following symbols: $a, b$-material constants for the inert; $c$-sound speed; $D$-Chapman-Jouguet detonation velocity; $e$-specific internal energy; $h$-Lagrangian coordinate, Eq. (4), $k-c_{1} / p_{1}{ }^{1 / 3}$, Eq. (8b); $P$-pressure; $t$-time; $u$-particle velocity; $U$-shock velocity; $v$-specific volume; $v=1 / \rho ; x$-distance; $x_{i 0}$-initial position of

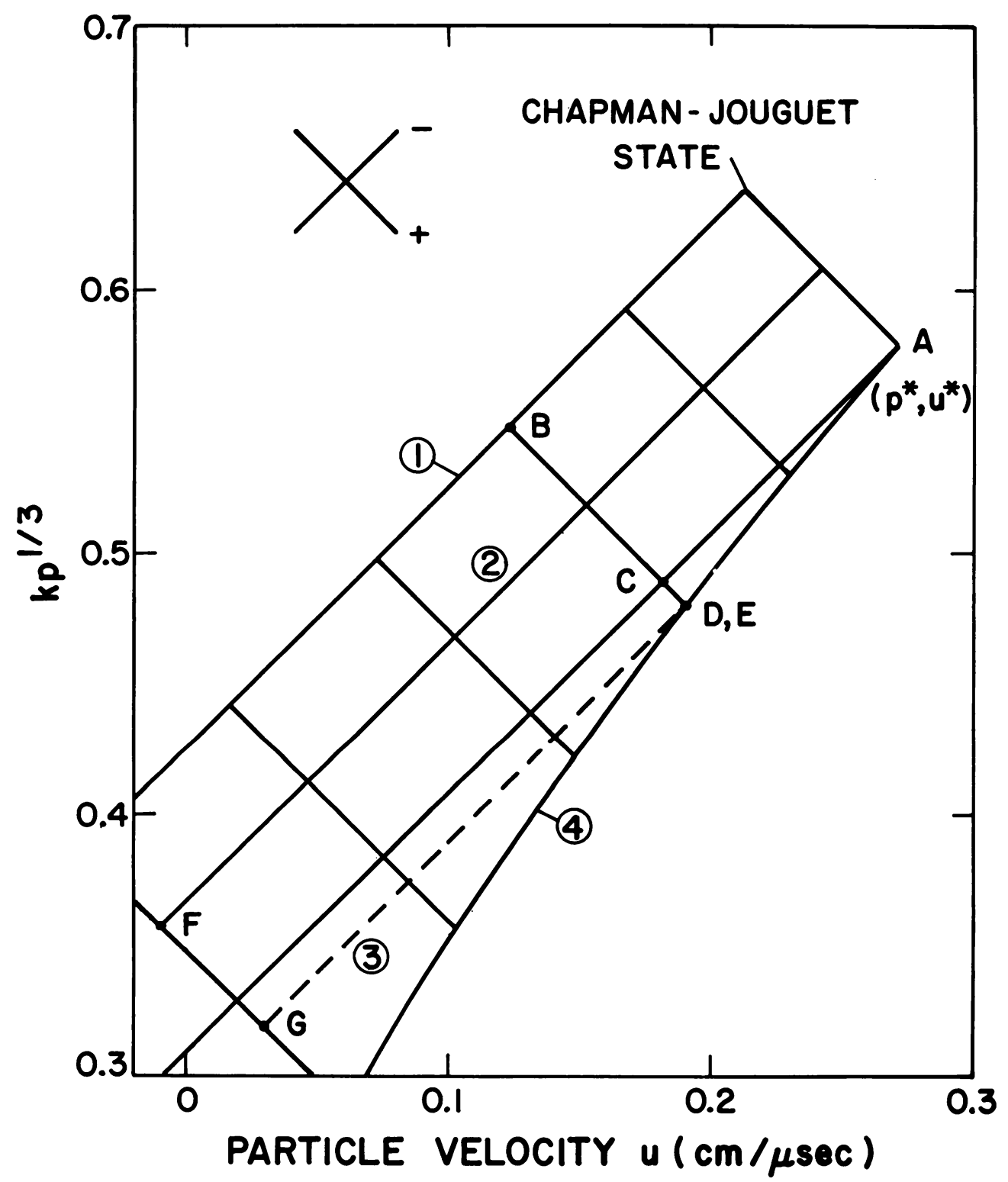

FIg. 2. The $p-u$ diagram. The characteristics in the explosive are straight lines by virtue of the function of pressure chosen for the ordinate which in the explosive is the sound speed (see Sec. 3). 


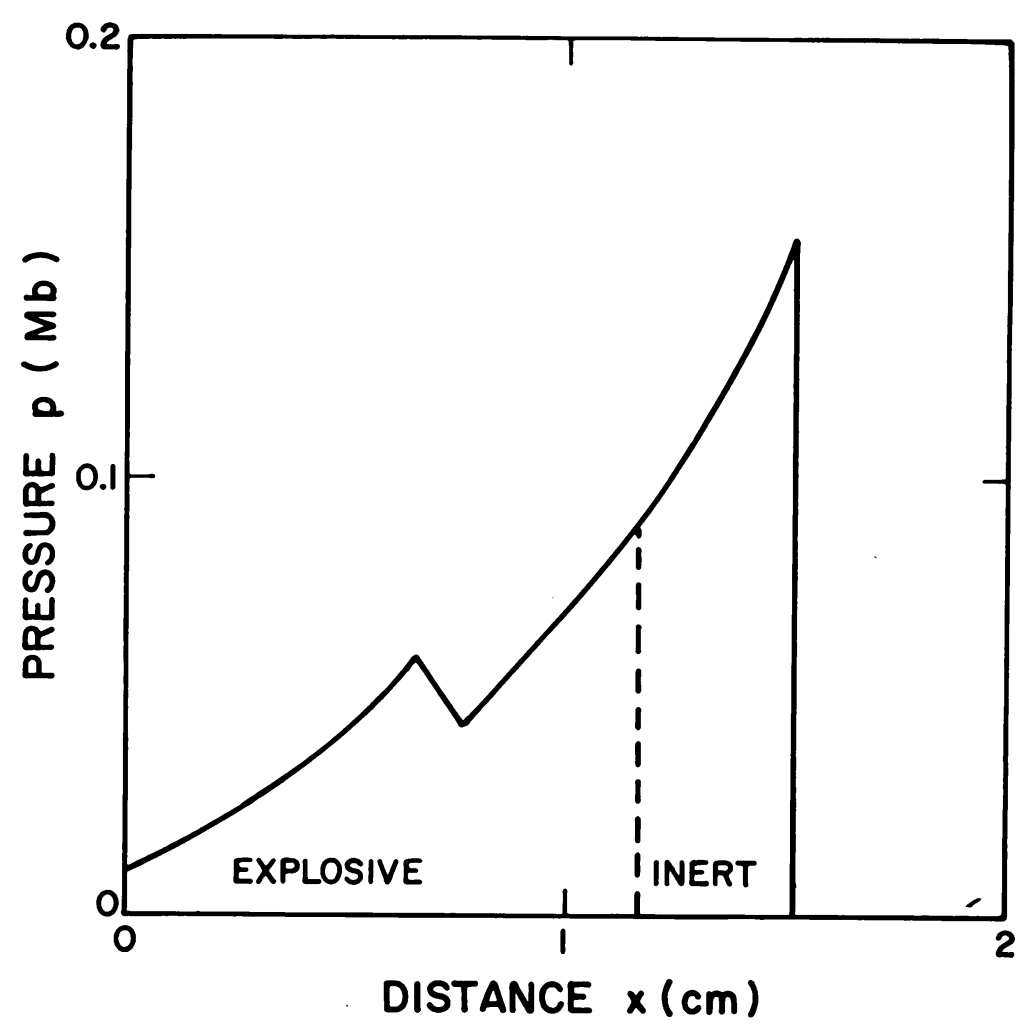

Fig. 3. The approximate pressure profile at $t=2 \mu \mathrm{s}$.

interface; $z$-acoustic impedance; $z \equiv \rho c ; \gamma$-material constant for the explosive; $\rho-$ density.

Sub- and superscripts are: 0 -initial; 1 -Chapman-Jouguet state of the explosive; *-state after initial wave interaction at interface; $i$-interface.

Literal subscripts denote partial differentiation. The units are $\mathrm{cm}-g-\mu \mathrm{s}$, with pressure in $\mathrm{Mb}$ and velocity in $\mathrm{cm} / \mu \mathrm{s}$.

The equations of motion are the inviscid (Euler) equations:

$$
\begin{gathered}
\rho_{t}+u \rho_{x}+\rho u_{x}=0, \\
u_{t}+u u_{x}+v p_{x}=0, \\
e_{t}+u e_{x}-p\left(v_{t}+u v_{x}\right)=0,
\end{gathered}
$$

together with the Rankine-Hugoniot relations across shocks:

$$
\begin{aligned}
\rho_{1} u_{1} & =\rho_{2} u_{2}, \\
\left(\rho u^{2}+p\right)_{1} & =\left(\rho u^{2}+p\right)_{2}, \\
\left(e+p v+\frac{1}{2} u^{2}\right)_{1} & =\left(e+p v+\frac{1}{2} u^{2}\right)_{2} .
\end{aligned}
$$

The subscripts 1 and 2 here refer, for these equations only, to the front and rear states of the shock. 
The equations of state are given in the form

$$
e=e(p, v) \text {. }
$$

We will find it convenient to define a Lagrangian coordinate $h$ in the inert:

$$
h(x)=\int_{0}^{x} \rho(x) d x ; \quad t=\text { constant }
$$

The characteristic relations are:

$$
\begin{aligned}
(d x / d t)_{ \pm} & =u \pm c \\
(d h / d t)_{ \pm} & = \pm z \\
(d p / d u)_{ \pm} & =\mp z
\end{aligned}
$$

where the plus and minus signs refer to the two families of characteristics, as shown in Figs. 1 and 2.

The detonation is treated under the usual simplifying assumptions. The reaction takes place instantaneously at the front. The detonation velocity (i.e., the velocity of the front) is constant at the Chapman-Jouguet value, following instantaneous initiation to this value at the left boundary. The Chapman-Jouguet detonation velocity is that velocity for which the flow immediately behind the front is sonic, i.e., for which $u+c=D$. We call the state at this point the Chapman-Jouguet state of the explosive and denote it by subscript 1 . From this front motion, it follows that the flow behind it is a simple rarefaction wave centered at $x=t=0$.

3. Equations of state. For the explosive, we choose the polytropic gas equation of state

$$
e=p v /(\gamma-1)
$$

with the value 3 for the constant $\gamma$. This makes all characteristics in $x-t$ space straight lines.

For the inert, we choose what we call, after its originator [5], the Walsh equation of state, described in the Appendix. Its key property is that for a shock followed by a rarefaction, as in the present problem, the Hugoniot locus and the rarefaction locus coincide in $p-u$ space, so that the acoustic impedance $z$ is a function only of the pressure, and the forward characteristics are straight lines in $h-t$ space. These properties make the "weak shock approximation" [4, sec. 74] exact and allow the deceleration of the shock to be calculated exactly, given the motion of the interface. They impart to the flow field in the inert enough of the properties of a simple wave to allow the exact determination of the interface motion provided the above equation of state (Eq. (6)) is used for the explosive.

4. Solution. We first collect the known flow properties in the two materials. In the explosive, the Chapman-Jouguet state is

$$
\begin{aligned}
p_{1} & =\rho_{0} D^{2} / 4, \\
v_{1} & =3 v_{0} / 4, \\
u_{1} & =D / 4, \\
c_{1} & =3 D / 4 .
\end{aligned}
$$


The entire flow in the explosive is isentropic, so that we have throughout it

$$
\begin{aligned}
v^{3} & =p_{1} v_{1}{ }^{3} / p, \\
c & =k p^{1 / 3} ; \quad k=c_{1} / p_{1}{ }^{1 / 3} .
\end{aligned}
$$

The plus and minus characteristics, straight lines throughout, are given by

$$
\begin{aligned}
& (d x / d t)_{+}=x / t=u+c=u+k p^{1 / 3}=\text { constant, } \\
& (d x / d t)_{-}=u-c=u-k p^{1 / 3}=\text { constant. }
\end{aligned}
$$

The constancy of $u+c$ along plus characteristics and of $u-c$ along minus characteristics is a consequence of the choice of the value of 3 for $\gamma$, the result for general $\gamma$ being

$$
u \pm 2 c /(\gamma-1)=\text { constant, } \quad c \propto p^{(\gamma-1) / 2 \gamma}
$$

In the inert, the entire flow lies, in $p-u$ space, on the Hugoniot (through the initial state) shown in Fig. 2, so that $p$ and functions of $p$ may be expressed in terms of $u$ :

$$
\begin{aligned}
p & =\rho_{0} u(a+b u), \\
z & =\rho c=d p / d u=\rho_{0}(a+2 b u) .
\end{aligned}
$$

At the shock, the shock velocity and particle velocity are related by

$$
U=a+b u,
$$

and the density is given by the mass conservation relation across the shock:

$$
\rho=\rho_{0} U /(U-u) .
$$

The plus characteristics in $h=t$ space are straight lines throughout, given by

$$
\left(h-h_{i}\right)=z\left(t-t_{i}\right),
$$

where $\left(h_{i}, t_{i}\right)$ is the point of intersection of the characteristic with the interface. Along each such characteristic $p, u$, and $z$ are constant.

The nice, i.e. isentropic, properties of the flow in the explosive, which we need for the exact solution, are spoiled by the presence of shocks. Thus we limit consideration at the outset to the undermatched case, in which the wave 2 reflected into the explosive from point $A$ of Fig. 1 is a rarefaction and not a shock. There remains another possibility of shock formation. To see this consider first the case shown in Figs. 1 and 2. Region 3 is the interaction region produced when the Taylor wave, after passing through wave 2 , produces a weak extended reflected wave as it passes through the interface. If, as in Fig. 2, the inert Hugoniot $A D \cdots$ lies entirely below the tail characteristic $A C \cdots$ of wave 2 , then the extended reflected wave, represented by the narrow triangular region 3 in Fig. 2, is a rarefaction (along arcs like $C D$ the pressure drops with time) and no shock will form. If, on the other hand, by changing the properties of the inert, its Hugoniot were made to lie above the tail characteristic of wave 2 , then the extended reflected wave would be a compression, and a shock would eventually form somewhere in it, probably on the tail of wave 2 . This would introduce small errors into our solution at very late times. The case shown is believed to be typical, but this point should be checked in any application.

We now proceed to obtain the prescription for the calculation of the entire flow. 
We first find the motions of the interface and shock, which must be obtained before the rest of the flow can be calculated.

4.1 Initial interaction. The starting point for both is the state produced by the initial interaction when the detonation front reaches the interface. The requirement that $p$ and $u$ be continuous across the interface gives as the solution point $A$ of Fig. 2, the intersection in $p-u$ space of the rarefaction locus (of negative slope through the Chapman-Jouguet point) for the explosive, and the shock Hugoniot through the initial state for the inert. The explosive rarefaction locus is given by Eq. (9a) with

$$
u+c=u_{1}+c_{1}=D,
$$

the second equality being the Chapman-Jouguet condition. The Hugoniot of the inert is given by Eq. (10a); elimination of $p$ from these two relations results in the desired equation for the initial particle velocity $u^{*}$ at the interface. It is the solution of

$$
D-u-k\left[\rho_{0} u(a+b u)\right]^{1 / 3}=0 .
$$

The pressure is given by Eq. (10a), the density in the explosive by Eq. (8a), and the density in the inert by Eqs. (10c) and (10d).

4.2 Interface. We obtain the interface motion in the form of a differential equation for $u$. Our starting point is the equation for the forward characteristics in the explosive, Eq. (9a), applied at the interface. We also use the relations between $p$ and $u$ and $z$ and $u$ in the inert, Eqs. (10). The special property that these hold throughout the inert and in particular at the interface is essential. There is also, of course, the continuity of $p$ and $u$ across the interface. Other quantities such as $\rho$ and $c$ are not continuous across the interface; where these enter, we must specify to which material they refer.

We first differentiate Eq. (9a) with respect to time along the interface to obtain

$$
d x / d t=u+c+(d u / d t+d c / d t) t,
$$

in which $c$ is the sound speed in the explosive given by Eq. (8b). Since the interface is a particle path, we also have on it

$$
d x / d t=u .
$$

We next express $d c / d t$ in (14) in terms of $d u / d t$. We can do this because $c$ is a function of $p$ alone and because $p$ at the interface is a function of $u$ alone by virtue of the inert relation (10a) applied at the interface. The result is

$$
\begin{aligned}
d c / d t & =(d c / d p)(d p / d u) d u / d t \\
& =\frac{1}{3} k p^{-2 / 3} z d u / d t,
\end{aligned}
$$

in which the substitution of $z$ for $d p / d u$ follows from (10b), applied, like (10a), at the interface, so that $z$ is the value of $\rho c$ in the inert. Substituting (17) and (15) into (14) and solving for $d u / d t$ gives the desired result

$$
\left.d u / d t=F(u, t)=-\left[t\left(1 / k p^{1 / 3}\right)+z / p\right)\right]^{-1},
$$

in which $p$ and $z$ are functions of $u$ by Eqs. (10). The position $x$ is given by Eq. (9a) as

$$
x=\left(u+k p^{1 / 3}\right) t .
$$

The initial conditions are: the value of $u$ from the interface match, given by Eq. (13), 
and the time at which the detonation reaches the interface,

$$
t=t^{*}=x_{i 0} / D \text {. }
$$

In the explosive, the density is given by Eq. (8a), and the sound speed by Eq. (8b). In the inert the density is given by the isentrope relation given in the Appendix (Eq. (A2a)) with $\hat{p}$ and $\hat{v}$ the values from the interface match; and the sound speed is given by $c=z / \rho$.

Eq. (18) can be integrated to

$$
\ln \left(t^{*} / t\right)=\left.\left[k^{-1} \rho_{0}{ }^{-1 / 3} a^{1 / 3} b^{-2 / 3} B_{u}(4 / 3,4 / 3)+\frac{1}{3} \ln u+\frac{1}{3} \ln \left(\rho_{0}(a+b u)\right)\right]\right|_{u^{*}} ^{u}
$$

where $B_{u}(4 / 3,4 / 3)$ is the incomplete beta function

$$
B_{u}(4 / 3,4 / 3)=\int_{0}^{u} x^{-1 / 3}(1-x)^{-1 / 3} d x
$$

4.3 Shock. We obtain the shock motion in the form of a differential equation for $u$, with time $t$ on the shock path as independent variable. The key property is that along a plus characteristic in the inert, such as $D E$ of Figs. 1 and 2, $p$ and $u$ (and therefore $z$ ) are constant, so that the values of $p$ and $u$ at $E$ are just those at $D$. The equation of such a characteristic in $h-t$ space is Eq. (11), in which $\left(h_{i}, t_{i}\right)$ is its intersection with the interface. To identify the $p, u$ states on the shock with their counterparts on the interface, we must determine $t_{i}$ as a function of $t$. We first write an equation for $d u / d t$ in terms of $d t_{i} / d t$. In the present context, the interface Eq. (18) becomes

$$
d u / d t_{i}=F\left(u, t_{i}\right),
$$

or

$$
d u / d t=F\left(u, t_{i}\right) d t_{i} / d t,
$$

which is the desired equation for $u$, provided we can express $d t_{i} / d t$ in terms of $u, t$, and $t_{i}$. To do this, we differentiate Eq. (11) with respect to $t$ to obtain

$$
d h / d t=\left(d z / d t_{i}\right)\left(d t_{i} / d t\right)\left(t-t_{i}\right)+z\left(1-d t_{i} / d t\right) .
$$

Now from the definition of $h$, Eq. (4), and from Eq. (10c) for $U$ we have

$$
d h / d t=\rho_{0} U=\rho_{0}(a+b u),
$$

and from Eqs. (10b) and (22a),

$$
d z / d t_{i}=2 \rho_{0} b F\left(u, t_{i}\right) .
$$

Making these substitutions in Eq. (23) and solving for $d t_{i} / d t$ gives the desired result:

$$
\begin{aligned}
d t_{i} / d t & =\rho_{0} b u / G\left(u, t, t_{i}\right), \\
G\left(u, t, t_{i}\right) & =z-2 \rho_{0} b F\left(u, t_{i}\right)\left(t-t_{i}\right) .
\end{aligned}
$$

This is to be integrated simultaneously with Eq. (22b). The initial conditions are the same as those for the interface. The shock position $x$ is given by Eq. (11), which with the definition of $h$ becomes

$$
x=x_{i 0}+z\left(t-t_{i}\right) / \rho_{0} .
$$


The shock velocity is given by Eq. (10c), the density by Eq. (10d), and the sound speed by Eq. (10b).

4.4 Particle paths in the inert. The derivation of these equations closely parallels that of those for the shock and will not be given here. The results are

$$
\begin{aligned}
d t_{i} / d t & =z / G\left(u, t, t_{i}\right), \\
d u / d t & =z F\left(u, t_{i}\right) / G\left(u, t, t_{i}\right), \\
d x / d t & =u .
\end{aligned}
$$

The initial conditions for the integration are those at the point where the particle enters the shock. The density may be obtained from the isentrope relations, Eq. (A2a).

4.5 Particle paths in the explosive. The particle path must be broken into three parts, corresponding to its traversal of regions 1, 2, and 3 of Fig. 1. The boundaries of region 2 are the head and tail characteristics of the centered rarefaction reflected from the match point:

$$
x / t=u_{1}-c_{1}, \quad x / t=u^{*}-c^{*} .
$$

Region 1. Here we have, from Eqs. (8) and (9),

$$
\begin{aligned}
& u+c=x / t \\
& u-c=u_{1}-c_{1}
\end{aligned}
$$

or, eliminating $c$,

$$
u=\frac{1}{2}\left(u_{1}-c_{1}\right)+\frac{1}{2} x / t
$$

The particle path equation is then

$$
d x / d t=u=\frac{1}{2}\left(u_{1}-c_{1}\right)+\frac{1}{2} x / t .
$$

The initial state for the integration is the position $x_{0}$ and time $t_{0}$ at which the particle enters the detonation front with

$$
t_{0}=x_{0} / D
$$

Eq. (30) may be integrated to

$$
\left[x / t-\left(u_{1}-c_{1}\right)\right]=\left[x_{0} / t_{0}-\left(u_{1}-c_{1}\right)\right]\left(t_{0} / t\right)^{1 / 2} .
$$

Values of $u$ and $c$ are obtained from Eqs. (29) and (28a), and $p$ and $v$ from Eqs. (8b) and (8a).

Region 2. Here we have, in similar fashion,

$$
\begin{aligned}
u+c & =x / t, \\
u-c & =\left(x-x_{i 0}\right) /\left(t-t^{*}\right), \\
u & =\frac{1}{2}\left(x-x_{i 0}\right) /\left(t-t^{*}\right)+\frac{1}{2} x / t, \\
d x / d t & =u=\frac{1}{2}\left(x-x_{i 0}\right) /\left(t-t^{*}\right)+\frac{1}{2} x / t .
\end{aligned}
$$

The negative characteristic here extends from a point such as $F$ (Fig. 1) to the match point $A$ at $\left(x_{i 0}, t^{*}\right)$. 
Region 3. Here we have a point such as $G$ of Fig. 1, with the minus characteristic intersecting the interface. The situation is similar to that for the shock path, in that the state at $G$ is related to that at $D$, where the minus characteristic intersects the interface. Here, however, the two states are not identical in $p$ and $u$, so the situation is more complicated. Let $x$ and $t$ be a point on the particle path, such as point $G$, and $x_{i}$ and $t_{i}$ be the point of intersection of the minus characteristic through it with the interface, point $D$. The characteristic relations are

$$
\begin{aligned}
u+c & =x / t, \\
u-c & =\left(x-x_{i}\right) /\left(t-t_{i}\right)=u_{i}-c_{i}, \\
u & =\frac{1}{2}\left[\left(x-x_{i}\right) /\left(t-t_{i}\right)+x / t\right],
\end{aligned}
$$

where $c_{i}$ is the sound speed in the explosive at the interface, given by Eq. (8b). As in the case of the shock, we obtain an equation for $d t_{i} / d t$. We begin by differentiating Eq. (35b) with respect to $t_{i}$ to obtain

$$
\begin{aligned}
(d x / d t)\left(d t / d t_{i}\right)-d x_{i} / d t_{i}=\left(d u_{i} / d t_{i}-d c_{i} / d t_{i}\right)\left(t-t_{i}\right) & \\
& +\left(u_{i}-c_{i}\right)\left(d t / d t_{i}-1\right) .
\end{aligned}
$$

Now $d x_{i} / d t_{i}$ and $d u_{i} / d t_{i}$ are already known from the interface solution, with $d c_{i} / d t$ given in terms of $d u_{i} / d t$ by Eq. (16). Using these results, together with the constancy of $u-c$ along negative characteristics, we find

$$
d t_{i} / d t=(x / t-u) /\left[k p_{i}{ }^{1 / 3}+F\left(u_{i}, t_{i}\right)\left(1-\frac{1}{3} k p_{i}{ }^{-2 / 3} z_{i}\right)\left(t-t_{i}\right)\right] .
$$

The remaining particle path equations are

$$
\begin{aligned}
d u_{i} / d t & =F\left(u_{i}, t_{i}\right) d t_{i} / d t, \\
d x / d t & =u, \\
x_{i} & =\left(u_{i}+k p_{i}{ }^{1 / 3}\right) t_{i},
\end{aligned}
$$

and Eq. (36a) for $u$. As before $p_{i}$ and $z_{i}$ are the functions of $u_{i}$ given by Eqs. (10).

5. Summary and numerical results. We have presented an "exact" (i.e., reduction to ordinary differential equations) solution of the one-dimensional time-dependent problem of the deceleration of the shock in an inert driven by a steady unsupported Chapman-Jouguet detonation, for special but realistic equations of state. An important restriction is that the solution applies only to the case in which the shock impedance of the inert is less than that of the explosive.

The motions of the interface and shock are of greatest interest. The interface motion is the solution of Eqs. (18) and (19) and the shock motion is the solution of Eqs. (22), (25), and (26). The initial conditions for both are given by Eqs. (13) and (20). Functions of $u$ appearing in the equations are given by Eq. (10).

We calculate the shock and interface for the explosive Composition B and the inert plexiglas. The constants used are

Composition B: $\quad \rho_{0}=1.6 \mathrm{~g} / \mathrm{cc}, \quad D=0.85 \mathrm{~cm} / \mu \mathrm{S}$

Plexiglas: $\quad \rho_{0}=1.19, \quad a=0.257, \quad b=1.54$. 
Composition $\mathrm{B}$ has a measured density of $1.714 \mathrm{~g} / \mathrm{cc}$ and a detonation velocity of $D=0.7991 \mathrm{~cm} / \mu \mathrm{s}$, with a Chapman-Jouguet pressure of $0.291 \mathrm{Mb}$ [6]. Although later work [7] indicates a significant uncertainty in the measurement of the Chapman-Jouguet pressure, we use the value for [6], which gives a Chapman-Jouguet state $\gamma$ of 2.77. With $\gamma=3$, we increase $D$ and decrease $\rho_{0}$ under the constraint of constant ChapmanJouguet pressure (Eq. (7a)) at the experimental value until we obtain the best match to the experimental isentrope [6] in $p-u$ space; the rounded values above match this curve almost within experimental error down to $1 \mathrm{~kb}$. This is of course done at the cost of increasing $D$ so that while the shock's initial pressure is correct, its rate of deceleration is slightly too large.

The measured plexiglas Hugoniot [8] is well represented by a linear $U-u$ relation (again we have rounded the constants). The approximation imposed by the Walsh equation of state may be judged by its prediction that the free-surface velocity produced by the shock impinging on a free surface (with resulting expansion to zero pressure) is always exactly twice the particle velocity at the shock; the experimental ratio for a shock pressure of $0.2 \mathrm{Mb}$ is 2.04 [8]. We remark that the coincidence between the Hugoniot and rarefaction locus in $p-u$ space, which is responsible for the ratio of exactly two

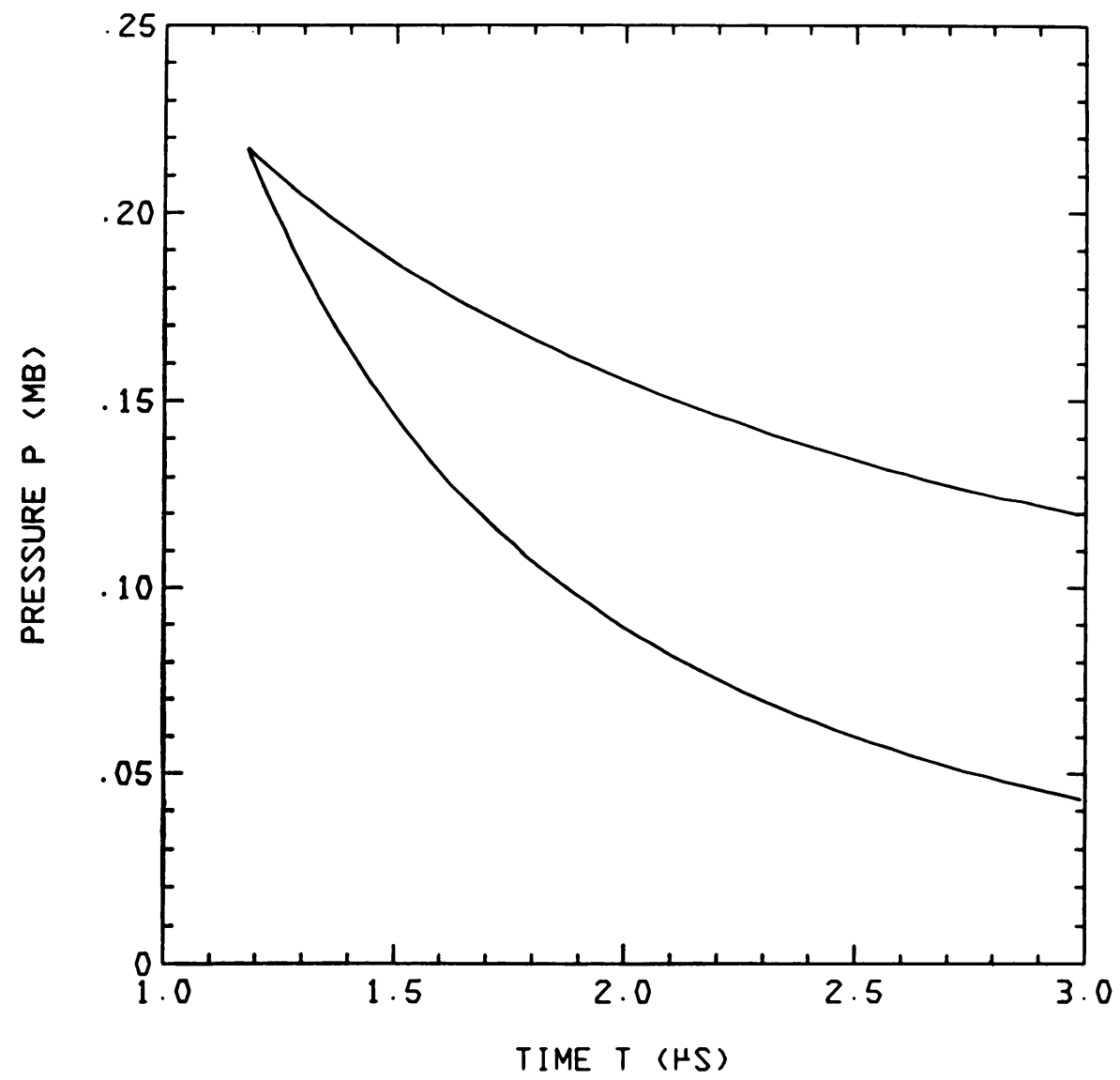

Fig. 4. Pressure at the interface (lower curve) and at the shock (upper curve). 
between the free-surface and shock-particle velocity, does not imply coincidence of these two curves in $p-v$ space.

The results of the calculation are given in Table 1 and Figs. 4 and 5. (Figs. 1 through 3 are also drawn from this problem.) Some idea of the entropy variation due to the shock deceleration may be had by comparing the densities and characteristic slopes at the two ends of a plus characteristic in the inert as given in Table 1. The characteristic through the shock path at $t=3$ originates at the interface at $t=1.69$. The constant pressure along the characteristic is $0.119 \mathrm{Mb}$, the interface particle having expanded to this value from the initial pressure of $0.216 \mathrm{Mb}$. The density at the shock is $1.81 \mathrm{cc} / \mathrm{g}$, $4 \%$ higher than at the interface, and the plus characteristic slope $u+c$ at the shock is $0.730 \mathrm{~cm} / \mu \mathrm{s}, 3 \%$ less than that at the interface.

Appendix A: The Walsh equation of state. The Walsh equation of state is constructed so that the relation between $p$ and $u$ in a rarefaction wave originating at any point on the shock Hugoniot centered at the normal initial state $\left(p=0, v=v_{0}\right)$ is the same as that for the shock. (The inert Hugoniot of Fig. 2 is an example.) The shock Hugoniot is taken as $U=a+b u$, which is a good representation of experimental data



FIg. 5. Particle velocity at the interface (lower curve) and at the shock (upper curve). 
TABLE 1.

Interface particle path and shock states for the explosive Composition $\mathrm{B}$ and inert plexiglas. The column headings are: $T, X, P, U, R H O, C$ for time $t$, position $x$, pressure $p$, particle velocity $u$, density $\rho$ (inert) and sound speed c (inert); RHOE, CE for density and sound speed in the explosive; UU for shock velocity $U$; and TI, XI, RHOI, CI for time $t_{i}$, position $x_{i}$ and inert density and sound speed at the point where the plus characteristic through the shock path at $(t, x)$ intersects the interface path. Units are $\mathrm{cm}-\mathrm{g}-\mu \mathrm{s}$, with velocity in $\mathrm{cm} / \mu \mathrm{S}$ and pressure in $\mathrm{Mb}\left(10^{11} \mathrm{~N} / \mathrm{m}^{2}\right)$.

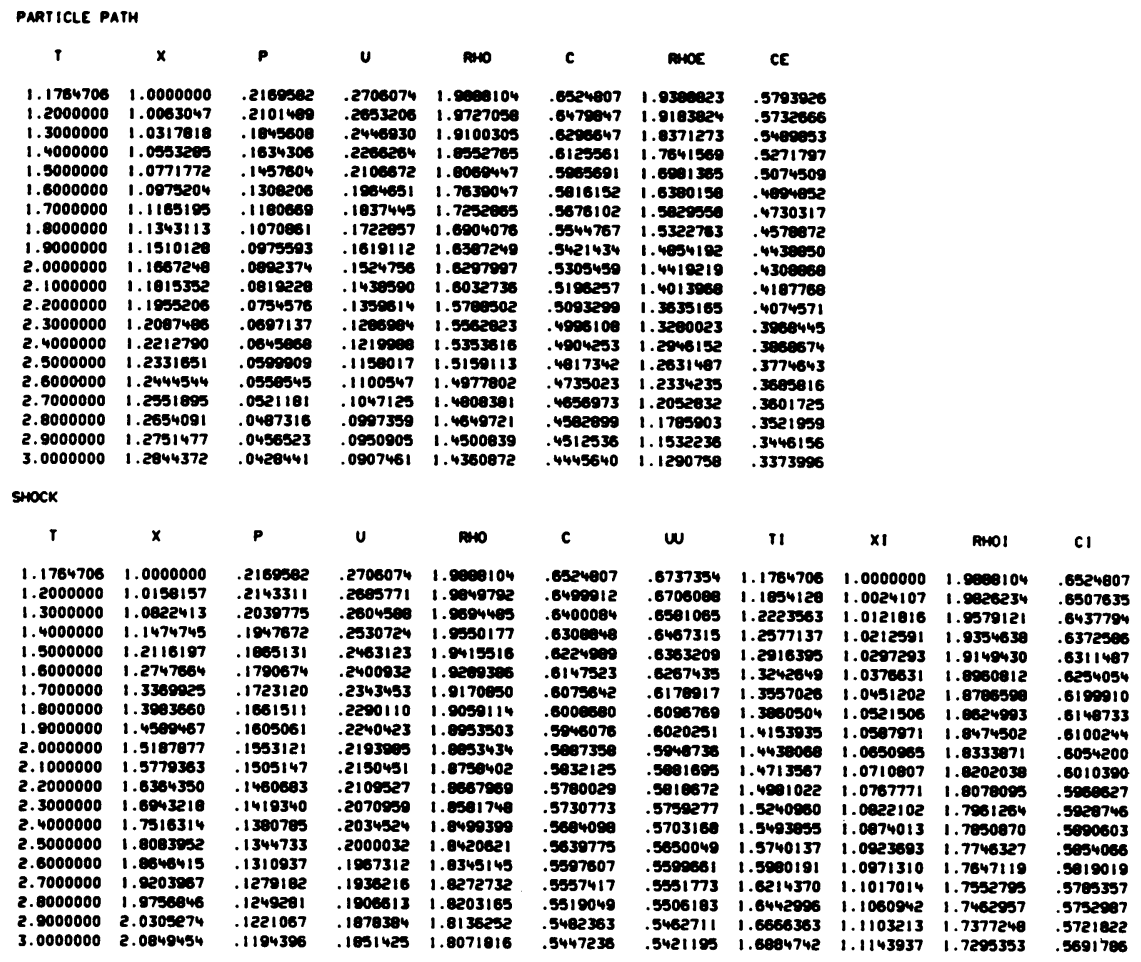

for many materials. The coincidence of the $p-u$ curves makes it possible to reduce the problem of the overtaking of a shock by a rarefaction wave to the solution of an ordinary differential equation.

This equation of state was first given by Walsh [5]. Some of its thermal properties were later explored by Enig [9]. We summarize the results here, and give a suggested computational algorithm for the function $p(v, e)$, which is required by most computer codes which treat the time-dependent problem.

The shock Hugoniot (caret) through the initial state is

$$
\begin{gathered}
\hat{p}=\rho_{0} a^{2} \eta /(1-b \eta)^{2}, \\
\hat{e}=\frac{1}{2} \eta \hat{p} / \rho_{0}, \\
\eta \equiv 1-\rho_{0} / \hat{\rho} .
\end{gathered}
$$

The isentrope through a point $(\hat{p}, \hat{v}, \hat{e})$ on the shock Hugoniot is

$$
p+\pi=(\hat{p}+\pi) e^{-\beta(v-\hat{t})},
$$




$$
\begin{gathered}
e=\hat{e}+(p-\hat{p}) / \beta-(\pi / \beta) \ln [(p+\pi) /(\hat{p}+\pi)] \\
\beta=4 \rho_{0} b, \quad \pi=\rho_{0}^{2} a^{2} / \beta .
\end{gathered}
$$

Most finite-difference computer codes require the equation of state in the form $p(e, v)$. We have done this as follows. First determine the point $(\hat{p}, \hat{v}, \hat{e})$ at which the isentrope through the given $e, v$ intersects the Hugoniot. This may be obtained by iterative solution of

$$
\tilde{e}(\hat{v})-e=0,
$$

for $\hat{v}$, with $e$ the given $e$, $\tilde{e}$ the isentrope function (A2b) with the given $v$, and $\hat{e}(\hat{v})$ and $\hat{p}(\hat{v})$ the Hugoniot functions (A1). With this value of $\hat{v}, p(v, e)$ is given by the isentrope function (A2a).

\section{REFERENCES}

[1] C. M. Ablow, Wave refraction at an interface, Quart. Appl. Math 18, 15-29 (1960)

[2] W. E. Drummond, Explosive induced shock waves: Part 1, plane shock waves, J. Appl. Phys. 28, 1437-41 (1957)

[3] V. N. Kondratev, I. V. Nemchinov, and B. D. Khristoforov, O zatukhanii v tverdom tele ploskikh udarnyth voln, vyzvannykh varyvom (On the attenuation in a solid of a plane shock wave generated by explosive), Zh. Prikl. Mekh. Tekh. Fiz. 4, 61-65 (1968)

[4] R. Courant and K. O. Friedrichs, Supersonic flow and shock waves, Interscience, New York, 1948

[5] J. M. Walsh, Shock attenuation for an arbitrary impluse, General Atomic Division of General Dynamics Corp. Report GAMD-2341, June 19, 1961

[6] W. E. Deal, Measurement of the reflected shock Hugoniot and isentrope for explosive reaction products, Phys. Fluids 1, 523-27 (1958)

[7] W. C. Davis and D. Venable, Pressure measurements for composition B-3, in Fifth symposium on detonation, ACR-184, U.S. Naval Ordnance Laboratory, White Oak, Maryland, pp. 13-21 (1970)

[8] R. Kinslow, ed., High-velocity impact phenomena, Academic Press, New York, 1970, p. 556

[9] J. W. Enig, A complete $E, P, V, T, S$ thermodynamic description of metals based on the $P, u$ mirrorimage approximation, J. Appl. Phys. 34, 746-54 (1963) 